\title{
Infiltrabilité et caractéristiques physiques de croûtes formées sur massifs d'agrégats initialement secs ou humides soumis à des pluies simulées
}

\author{
JC Fiès ${ }^{1 *}$, T Panini $^{2}$ \\ 1 INRA, unité de science du sol d'Avignon, site Agroparc, F 84914 Avignon cedex 9, France; \\ 2 Istituto Sperimentale per lo Studio e la Difesa del Suolo, piazza d'Azeglio 30, 150121 Florence, Italie
}

(Reçu le 20 mai 1994; accepté le 2 juin 1995)

\begin{abstract}
Résumé - La formation de croûte sous l'effet des pluies entraîne la réduction de l'infiltrabilité des sols travaillés. La réalisation de ce phénomène est cependant en grande partie conditionnée par la constitution du sol et son état hydrique initial. Le rôle de ces 2 facteurs est étudié au laboratoire. Des massifs d'agrégats d'un sol limoneux $L$ et d'un sol argilo-limoneux AL, à l'état initial sec ou humide, sont soumis à des pluies $\left(26 \mathrm{~mm} / \mathrm{h}, 23 \mathrm{~J} / \mathrm{m}^{2} / \mathrm{mm}\right)$. Entre ces pluies sont intercalés des apports d'eau importants $(120 \mathrm{~mm} / \mathrm{h}, 3 \mathrm{~J} / \mathrm{m} 2 / \mathrm{mm})$ qui ont permis de mesurer la réduction de l'infiltrabilité des massifs avant qu'elle ne devienne perceptible par les mesures d'infiltration sous pluie. Avec les sols L-sec et AL-sec ainsi que L-humide, les agrégats se fragmentent. Sur sol AL-humide, il y a abrasion des agrégats. La réduction d'infiltrabilité qui accompagne la formation des croûtes se manifeste après une quantité de pluie plus importante sur sol inititalement humide que sur sol initialement sec, en particulier pour le sol AL. Dans tous les cas, la réduction de l'infiltrabilité se poursuit ensuite plus lentement sous l'effet des pluies. Le classement suivant des valeurs finales d'infiltrabilité est obtenu : $A L$-humide $>L-s e c=L-$ humide $>A L-$ sec. L'analyse de la porosité, basée sur la porosimétrie au mercure et les mesures de volume massique et de teneur en eau aux potentiels de $-0,1$ à $-10 \mathrm{~m}$, montre qu'une porosité structurale existe dans les croûtes. Le classement final de l'infiltrabilité est interprété selon 3 hypothèses : les pores structuraux interconnectés assurent le transfert de l'eau sous pluie ( $A L$ - humide), ou bien ils se désaturent entre les pluies et de l'air piégé les rend alors inefficaces (AL-sec), enfin, les pores structuraux sont saturés mais ne sont pas interconnectés (L-sec, L-humide).
\end{abstract}

infiltrabilité / croûte / porosimétrie au mercure / rétention en eau / pluie simulée

Summary - Infiltrability and measurement of physical characteristics of crusts developed on wet or dry soil aggregates under simulated rains. The sealing of a soil surface under rain reduces the infiltrability of cultivated soils. This phenomenon is largely conditioned by the soil constitution and the initital water content. These 2 factors were studied in the laboratory using a bed of soil aggregates. Silty $(L)$ and a clay-silty $(A L)$ soils were chosen. Soils were initially dry or wet and then received a series of rains $\left(26 \mathrm{~mm} / \mathrm{h}, 23 \mathrm{~J} / \mathrm{m}^{2} / \mathrm{mm}\right)$. Between 2 subsequent rains, the infiltrability was measured under a large amount of water $\left(120 \mathrm{~mm} / \mathrm{h}, 3 \mathrm{~J} / \mathrm{m}^{2} / \mathrm{mm}\right)$, in order to demonstrate that the reduction in the infiltrability occurs before the phenomenon can be actually measured under rain. Aggregates of the dry $-L$, dry $A L$ and wet $-L$ soils break into fragments. The wet $-A L$ soil shows abrasion of aggregates. The reduction in soil infiltrability, which is related to crust formation, appears after a greater quantity of rains if the soil was initially wet than when it was initially dry. The decrease in infiltrability then proceeds slowly in all cases, and the final infiltrability ranking is: wet $A L>d r y-L=$ wet $-A L>d r y-L$. The porosity of the crust was analysed using mercury porosimetry, bulk volume measurements and the volumetric water contents at the pressure potentials ranging from -0.1 to $-10 \mathrm{~m}$. The crust 
porosity analysis shows that a structural porosity exists within the crusts. Three hypothesis are made to explain the final infiltrability order: interconnected structural pores perform the water transfer under rain (AL - wet); air can be caught in structural pores due to desaturation between subsequents rains (dry - AL); or structural pores remain saturated but are not interconnected (dry $-L$ and wet $-L$ ).

\section{infiltrability / crust / mercury porosimetry / water retention / simulated rain}

\section{INTRODUCTION}

Dans les sols cultivés, le développement d'une croûte en surface entraîne généralement une réduction de l'infiltrabilité de l'interface sol-atmosphère (Mclntyre, 1958 ; Boiffin et Monnier, 1985). Cette réduction est consécutive à la fermeture de l'espace poral entre mottes et agrégats par les fragments et particules produits sous l'effet de différents mécanismes de désagrégation (Cernuda et al, 1954 ; Hénin et Monnier, 1956 ; Kheyrabi et Monnier, 1968) et sous l'effet du tassement (Govers et Poesen, 1985 ; Slattery et Bryan, 1992).

Dans le cas de sols moyennement argileux et calciques, l'éclatement et l'abrasion constituent les mécanismes dominants de désagrégation et déterminent la nature et la taille des éléments résultant (Le Bissonnais, 1988 ; Le Souder et al, 1991). Mais parce que la dominance de l'éclatement ou de l'abrasion dépend du degré de saturation (Boiffin, 1984 ; Le Bissonnais, 1988), l'état hydrique de surface va alors conditionner la morphologie des croûtes (Valentin et Bresson, 1992). Ainsi, Bresson et Cadot (1992) montrent que, pour les sols limoneux, un état initial sec favorise l'éclatement des agrégats et conduit à la formation d'une croûte par accumulation de fragments terreux. En revanche, quand les agrégats sont initialement saturés, il se forme alors une croûte d'illuviation. Dans ce cas, l'abrasion progressive des agrégats sous l'impact des gouttes libère des particules fines qui viennent combler l'espace poral inter-agrégats. Ces particules sont surtout des particules élémentaires (Le Bissonnais et al, 1989).

Ces croûtes d'origines différentes devraient présenter des niveaux différents d'infiltrabilité. Bien que nombreux cependant, les travaux expérimentaux reliant le développement d'une croûte superficielle à la réduction de l'infiltrabilité portent soit sur des sols initialement secs (Mclntyre, 1958 ; Freebairn et al, 1991) soit sur des sols humectés (Ben-Hur et Shainberg, 1989 ; Keren, 1989), mais les effets induits par la différence d'état hydrique initial ne sont pas étudiés. Pourtant, un ensemble d'observations tend à montrer que des agrégats préalablement saturés en eau sont plus résistants à l'action de la pluie (Boiffin et al, 1990 ; Truman et al, 1990) et que le processus de développement de la croûte est alors très lent (Le Bissonnais et al, 1989). Cela suggère qu'en climat tempéré, sur sol sensible à la battance et préparé pour un semis, des conditions hydriques différentes en surface entraîneraient des différences dans la quantité de pluie nécessaire à la formation de la surface encroûtée et dans le régime d'abaissement de l'infiltrabilité.

Nous avons choisi de vérifier ces hypothèses en étudiant l'effet de l'état hydrique initial sur l'évolution de l'infiltrabilité de massifs d'agrégats soumis à des pluies simulées. L'utilisation d'agrégats facilite les actions de dégradation homogènes par la pluie. De plus, on sait depuis les travaux de Boiffin (1985) que l'encroûtement des sols travaillés se développe d'abord dans les aires occupées par les fragments terreux de petite dimension. C'est seulement dans ces aires que l'infiltrabilité commence à diminuer de manière importante. Dans le cas de fragments isodimensionnels, Freebairn et al (1991) ont montré qu'avec des petites mottes une plus forte quantité de pluie est nécessaire pour obtenir un développement de la croûte et une infiltrabilité finale comparables à ceux atteints avec des agrégats.

Dans cette étude 2 aspects ont été particulièrement développés. Le premier concerne la mesure de l'infiltrabilité avant apparition du flaquage sous pluie. En effet, la mesure de l'infiltrabilité caractérise le plus souvent un état physique déjà suffisamment modifié pour que l'infiltrabilité soit devenue inférieure à l'intensité de la pluie et qu'il y ait flaquage et ruissellement. Si l'on peut considérer que la mesure de l'infiltrabilité avant flaquage peut être négligée quand les modifications physiques se produisent très rapidement au début d'une pluie, elle devient nécessaire quand ces modifications se produisent lentement au cours d'une ou de plusieurs pluies. Cette mesure d'infiltrabilité sera appelée par la suite mesure sous apport d'eau important.

Le second aspect porte sur la caractérisation de l'espace poral des croûtes en relation avec 
leur niveau d'infiltrabilité. On utilise ici les notions d'espace poral textural, ie déterminé par l'organisation des particules élémentaires (Childs, 1969 ; Monnier et al, 1973) et d'espace poral structural, ie résultant de l'assemblage des agrégats et de leurs fragments (Stengel, 1979). L'interprétation sera basée sur la comparaison entre les résultats obtenus sur agrégats, qui représentent la référence texturale et les résultats obtenus sur échantillons de croûtes.

\section{MATÉRIEL ET MÉTHODES}

\section{Sols}

Deux sols de sensibilité différente à l'action de l'eau ont été choisis en se basant sur les relations entre stabilité structurale et constitution des sols (Monnier, 1984). Ces 2 sols ont été prélevés dans la couche $0-20 \mathrm{~cm}$ de parcelles cultivées. Celui que nous appellerons le sol $\mathrm{L}$ est de texture limoneuse et se classe d'après sa constitution dans la famille des sols très instables. II s'agit d'un Gleyic Luvisol (classification FAO) prélevé à Marçon (Sarthe). Le second sol, qui sera dénommé par la suite sol $A L$, est de texture argilo-limoneuse et appartient à la famille des sols à stabilité médiocre. C'est un Eutric Cambisol (classification FAO), à phase caillouteuse, recarbonaté, prélevé à Burelles (Thiérache). Leurs caractéristiques sont données au tableau I. Leur capacité d'échange est saturée en $\mathrm{Ca}^{2+}$.

\section{Dispositif}

Le dispositif (fig 1) permet en particulier de travailler à succion imposée (De Roo et Riezebos, 1992). Le cylindre transparent de $0,04 \mathrm{~m}^{2}$ de section reçoit $0,8 \mathrm{~kg}$ d'agrégats de diamètre $2-3 \mathrm{~mm}$ qui forment une couche d'épaisseur $20 \mathrm{~mm}$ sur une couche de $1,8 \mathrm{~kg}$ de sable épaisse de $25 \mathrm{~mm}$. La couche de sable, mise en place dans de l'eau en excès, est soumise à une succion de $-0,4 \mathrm{~m}$, obtenue en disposant un bac de drainage en position basse. Cette succion est maintenue en permanence pendant la mise en place des agrégats et durant les pluies. La couche de sable a un taux de saturation de $99 \%$ pour la succion imposée et son point d'entrée d'air est atteint à une succion de $-1 \mathrm{~m}$. Son infiltrabilité est voisine de $150 \mathrm{~mm} / \mathrm{h}$ et cette valeur limitera donc l'intensité maximale utilisable pour les apports d'eau importants, comme on le verra par la suite. Une inclinaison de $3 \%$ donnée au cylindre permet l'évacuation de l'excès d'eau quand il se forme en surface, par une ouverture de $15 \mathrm{~mm}$. Sous les apports d'eau importants, la hauteur de la lame d'eau observée au travers du cylindre au voisinage de l'ouverture n'excède pas $2 \mathrm{~mm}$.

\section{État hydrique initial des agrégats}

Deux états hydriques initiaux sont réalisés, correspondant à un état sec et un état humide que l'on désignera aussi par les termes de sol sec et de sol humide. Dans l'état sec, le dispositif est soumis immédiatement à la pluie après que l'on a rapidement disposé les

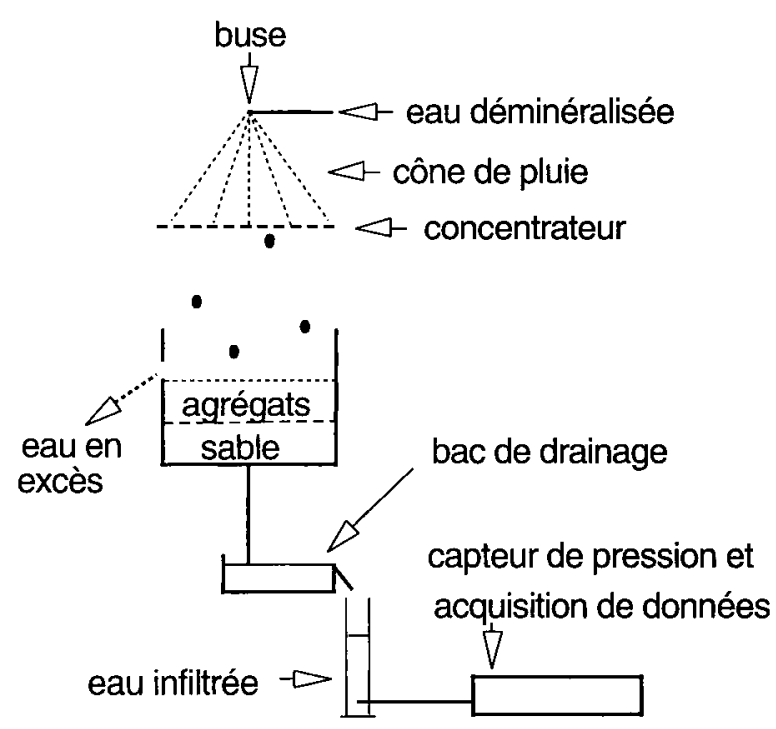

Fig 1. Dispositif de fabrication de croûtes et de mesures d'infiltration sous pluie et sous apport d'eau important.

Tableau I. Caractéristiques physiques des sols.

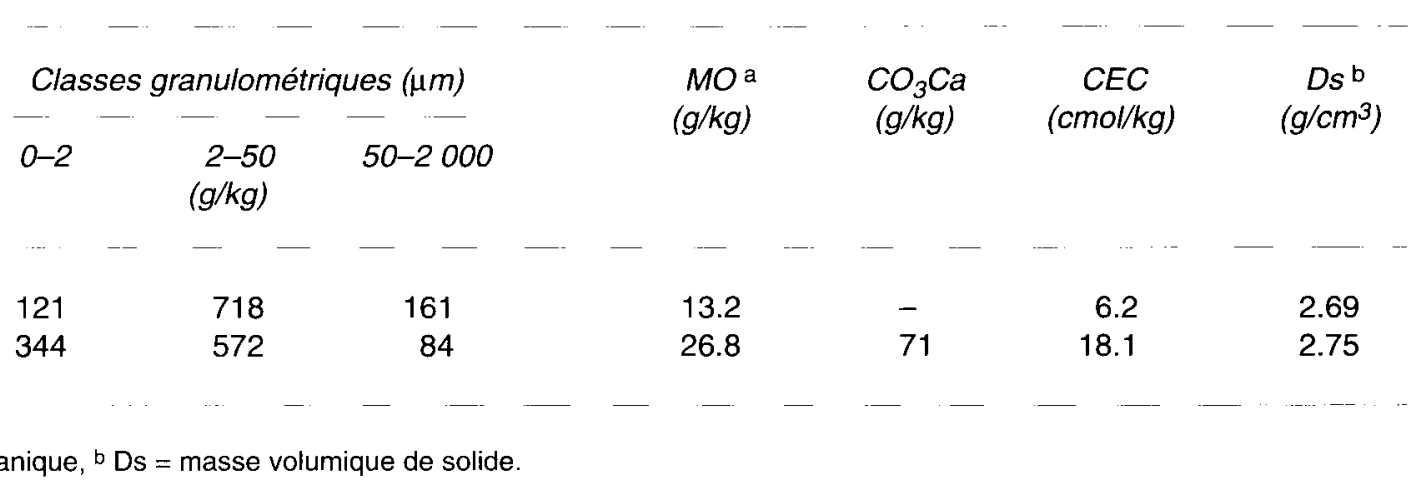

${ }^{a} \mathrm{MO}=$ matière organique,${ }^{b} \mathrm{Ds}=$ masse volumique de solide. 
agrégats secs à l'air sur le sable humide. Dans l'état humide, on laisse les agrégats s'humecter sous succion $(-0,4 \mathrm{~m})$, ce qui assure l'absence totale de fissuration à I'humectation (Stengel et Bourlet, 1987). Après $2 \mathrm{j}$, le bac est progressivement élevé et on laisse pendant quelques heures l'eau affleurer à la surface des agrégats. Enfin, le bac est ramené à sa position basse initiale et on attend une journée avant d'effectuer la première pluie.

L'observation de la couche, en surface et latéralement, montre que les agrégats ont conservé leur forme et ne se sont pas fragmentés. Des mesures de teneur en eau montrent que les agrégats restent saturés et que l'espace inter-agrégats est désaturé.

\section{Pluies simulées}

Le dispositif reçoit une succession de pluies. La durée de chaque pluie est modulée par les modifications survenant en surface et leur développement. Elle est de 7 à $20 \mathrm{~min}$ en début d'expérience et de 30 à $90 \mathrm{~min}$ ensuite.

Les pluies sont d'une intensité de $26 \pm 2 \mathrm{~mm} / \mathrm{h}$ et leur énergie cinétique moyenne est de $23 \mathrm{~J} / \mathrm{m}^{2} / \mathrm{mm}$. Elles sont réalisées avec de l'eau déminéralisée passant dans une buse à cône plein à une pression de $2 \mathrm{~b}$ (Poesen et al, 1990 ; Panini et al, 1993). Le jet de gouttelettes est ici intercepté par un concentrateur (fig 1) donnant des gouttes régulières tombant de $3 \mathrm{~m}$ de haut. Ce concentrateur est une plaque de $0,5 \mathrm{~m}$ de côté, percée tous les $20 \mathrm{~mm}$ de cônes d'où l'eau s'écoule en formant des gouttes de diamètre 5,3 \pm $0,3 \mathrm{~mm}$. La masse moyenne d'une goutte a été définie en pesant un nombre connu de gouttes prélevées à la base de 10 cônes pris au hasard et le diamètre moyen des gouttes a été calculé en admettant que les gouttes étaient sphériques. L'énergie cinétique des gouttes a été calculée à partir des données empiriques fournies par Epema et Riezebos (1983).

L'eau infiltrée est recueillie à la sortie du bac de drainage dans un tube cylindrique relié à un capteur de pression (fig 1). Les caractéristiques de ce capteur sont données par Tamari et al (1993). La pression hydrostatique à la base du tube est enregistrée toutes les $20 \mathrm{~s}$. Ce dispositif permet la mesure des quantités d'eau infiltrées sous pluie et lors des mesures d'infiltrabilité sous apport d'eau important.

Dans ces conditions, 2 groupes d'expériences ont été réalisés afin de permettre des prélèvements d'échantillons. Ils correspondent en moyenne à une somme de pluies de $69 \mathrm{~mm}$, soit $1560 \mathrm{~J} / \mathrm{m}^{2}$ pour le premier et de $225 \mathrm{~mm}$, soit $5165 \mathrm{~J} / \mathrm{m}^{2}$ pour le second.

\section{Mesure de l'infiltrabilité sous apport d'eau important}

Cette mesure vise à caractériser les niveaux élevés de l'infiltrabilité, en perturbant le moins possible l'état phy- sique du sol. Pour cela l'apport d'eau doit avoir une intensité élevée pour entraîner un excès d'eau en surface et une faible énergie cinétique. Ces premières conditions sont approchées en apportant l'eau directement par 2 buses semblables à celle déjà décrite, situées à $0,5 \mathrm{~m}$ de la surface. L'énergie cinétique de l'apport dans ces conditions est inférieure à $3 \mathrm{~J} / \mathrm{m}^{2} / \mathrm{mm}$. L'intensité de l'apport est de $120 \pm$ $10 \mathrm{~mm} / \mathrm{h}$, de manière à ne pas excéder la capacité de transfert de la couche de sable, et sa durée est limitée à $10 \mathrm{~min}$. Enfin, l'apport d'eau est réalisé après une première pluie pour minimiser les risques d'éclatement des agrégats. II faut noter qu'une telle mesure n'est possible qu'en l'absence de phénomène de dispersion. Pour les sols choisis, le fait que la capacité d'échange soit saturée en $\mathrm{Ca}^{2+}$ concourt au maintien de la stabilité du sol en condition de saturation.

L'enregistrement des quantités infiltrées sous apport d'eau important montre qu'un régime stationnaire s'établit en quelques minutes. Dans ce qui suit, chaque mesure sous apport d'eau important sera représentée par la moyenne des valeurs mesurées.

\section{Surface encroûtée}

Le taux de surface encroûtée est obtenu à partir de photographies de la surface prises à la suite de chaque pluie. Ces surfaces sont définies par l'absence de pore d'arrangement visible à l'œil entre agrégats ou fragments terreux. Sur les photographies, ces surfaces sont découpées et pesées pour définir leur aire.

\section{Porosimétrie au mercure}

La mesure a porté sur des échantillons de croûtes de 2 à $3 \mathrm{~mm}$ d'épaisseur et de $15 \mathrm{~mm}$ environ de côté et des agrégats. II n'y a pas eu de répétition. La porosimétrie au mercure permet de mesurer le volume total des pores dont les diamètres d'entrée se situent entre 400 et $0,006 \mu \mathrm{m}$ (Bruand et Prost, 1987). La mesure des quantités injectées $\Delta V$ dans l'intervalle de diamètre de pore $\Delta D$, permet aussi d'établir la courbe de densité de distribution volumétrique des pores (Renault, 1988). Cette courbe sera appelée courbe de distribution. Elle est calculée avec la relation $\Delta V / \Delta\left(\log _{10} D\right)$, approximation de la dérivée de la courbe cumulative d'intrusion (Fiès et Zimmer, 1981).

On comparera les courbes obtenues sur croûtes à celles des agrégats. La courbe de distribution obtenue sur agrégats permet de distinguer les 2 compartiments de la porosité texturale : d'une part les pores lacunaires dus à l'assemblage des grains du squelette (limon et sable) enrobés d'argile, d'autre part les pores de l'argile dus à l'assemblage des particules d'argile entre elles (Fiès et Bruand, 1990). Les pores lacunaires ont des caractéristiques qui dépendent du squelette et du taux d'argile. Leur volume total et le diamètre de leurs pores d'accès décroissent d'une part quand la taille du squelette diminue, d'autre part quand 
la teneur en argile augmente (Fiès, 1992). Les pores de l'argile ont un diamètre généralement inférieur à celui des pores lacunaires.

\section{Rétention en eau et volume massique des croûtes}

Sur des échantillons de croûte prélevés et préparés comme ci-dessus, les teneurs en eau sont mesurées pour 6 équilibres de potentiel allant de $-0,1$ à $-10 \mathrm{~m}$, avec 5 répétitions. L'écart type moyen de la teneur en eau $(\mathrm{g} / 100 \mathrm{~g})$ est de 0,27 pour les agrégats et de 0,55 pour les croûtes.

Le volume massique à ces équilibres de potentiel est mesuré en utilisant la pesée hydrostatique dans le pétrole (Monnier et al, 1973). Avec 5 répétitions, l'écart type du volume massique $\left(\mathrm{cm}^{3} / \mathrm{g}\right)$, est de 0,005 sur les agrégats et de 0,008 sur les croûtes.

Pour ces mesures à l'état humide, les croûtes sont d'abord réhumectées de manière ménagée sur table de succion ou dans une presse de Richards et amenées à potentiel nul.

L'ensemble de ces caractérisations physiques est aussi réalisé sur les agrégats initiaux, en s'attachant à définir leur niveau intrinsèque : lors de la réalisation des équilibres de potentiel par exemple, les agrégats sont disposés en couche lâche et mince et leur humectation à saturation est réalisée sans éclatement.

\section{Image de profil de croûte}

Des prélèvements destinés à l'observation du profil de la croûte et des agrégats sous-jacents sont imprégnés par une résine polyester contenant un azurant optique (Hartmann et al, 1992). Sur les sections polies, l'espace poral est mis en évidence en éclairage ultra violet
(360 nm), les surfaces occupées par la résine émettant une fluorescence bleue. Les images sont obtenues en plaçant un filtre (Kodak Wratten 2B) devant l'objectif photographique.

\section{RÉSULTATS ET DISCUSSION}

\section{Formation et extension de la croûte}

Une chute de $2 \mathrm{~mm}$ de pluie correspond à une couverture de toute la surface par les zones d'impact. Cependant les quantités de pluie nécessaires à l'apparition et à l'extension complète de la surface encroûtée varient selon l'état hydrique initial et la composition du sol (fig 2). Pour L-sec et $A L-s e c$, la formation de la croûte débute entre 2 et $4 \mathrm{~mm}$ de pluie et son extension s'achève avec $20 \mathrm{~mm}$ de pluie. La formation de la croûte ne débute qu'après une pluie de $13 \mathrm{~mm}$ pour Lhumide, de $41 \mathrm{~mm}$ pour AL-humide. Son extension est achevée avec $40 \mathrm{~mm}$ de pluie sur Lhumide et avec une quantité de pluie 5 fois supérieure sur AL-humide.

Un ensemble de modifications accompagne la formation de la croûte : des cratères apparaissent aux points d'impact des gouttes, les agrégats se fragmentent ou s'usent.

Ces cratères, de $15 \mathrm{~mm}$ de diamètre environ, ont un bord relevé qui confère un relief marqué à la surface. Leur manifestation précède de quelques millimètres de pluie la formation des premières surfaces encroûtées. Elle précède aussi la projection de fragments ou d'agrégats et le premier flaquage sous pluie.

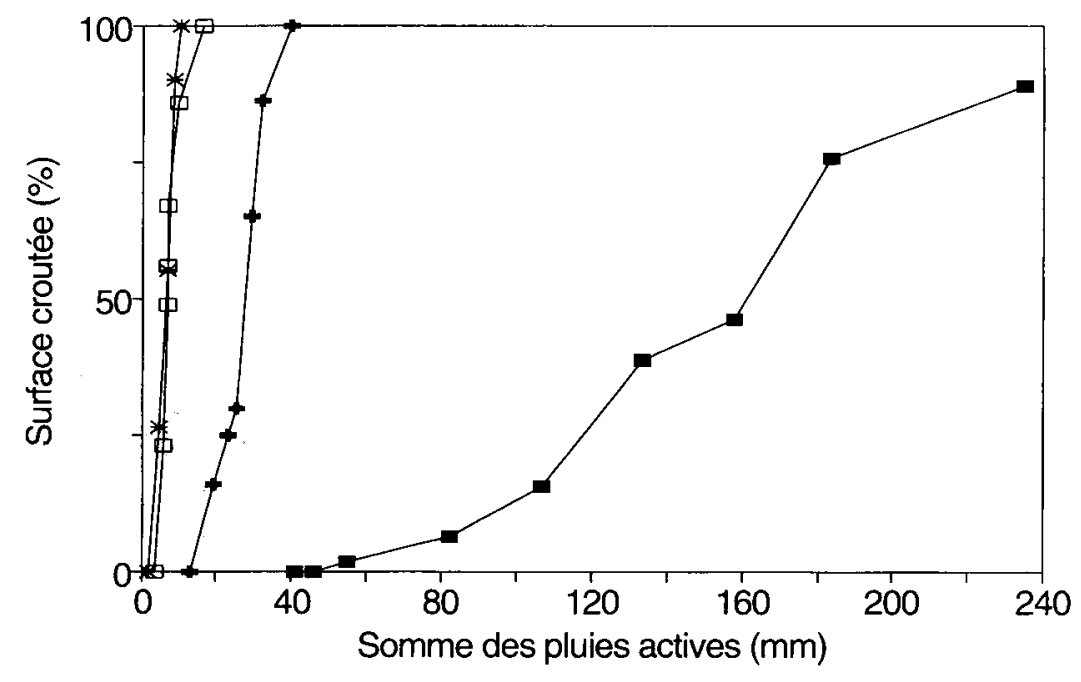

$\rightarrow$ L-sec $\quad \because$ AL-sec $\rightarrow$ L-humide $\rightarrow$ AL-humide
Fig 2. Taux d'occupation de la surface par une croûte en fonction de la pluie. $-x-L-s e c ;-7-A L-$ sec ; -+- L-humide ; - - ALhumide. 
Au niveau des agrégats, la désagrégation se signale de 2 manières : les agrégats apparaissent soit fragmentés aussitôt après l'impact d'une goutte soit progressivement arrondis. La fragmentation est toujours observée pour l'état sec. Elle est également observée pour le sol L-humide. Dans tous les cas, la fragmentation est synchrone avec la formation de cratères et les zones encroûtées apparaissent dans les cratères. Pour le sol AL-humide, les gouttes provoquent d'abord un déplacement brutal et limité d'agrégats, la surface restant inchangée. Puis se forment les cratères, aux parois constituées d'agrégats quasi entiers et parfois arrondis, mobilisés à chaque impact. Leur fond se présente comme un pavage d'agrégats arrondis réunis par une phase claire de particules fines. La réalisation de ce pavage représente le début de l'encroûtement observable.

D'après les travaux de Al-Durrah et Bradford (1982), la formation des cratères est due à la force radiale d'écoulement produite par chaque goutte d'eau après l'impact, qui provoque à la surface du sol la formation de cavités, en particulier si le niveau de cohésion est bas. La surface étant ici formée d'une couche meuble, la cohésion qui intervient n'est pas intrinsèque aux agrégats mais dépend de leur force de liaison. Sharma et Gupta (1989) ont montré, sur sable humide, que cette force dépend bien du potentiel de l'eau entre les grains, l'énergie nécessaire au détachement des grains par une goutte d'eau étant plus élevée au potentiel initial de $-0,15 \mathrm{~m}$ qu'avec celui de $-0,10 \mathrm{~m}$. La formation de cratères indique donc que le potentiel s'élève au voisinage de la surface. Cela implique une diminution de l'infiltrabilité et donc du transfert d'eau lors des impacts de gouttes suffisante pour que s'établissent et se maintiennent les conditions de potentiel propices à la formation de cratères.
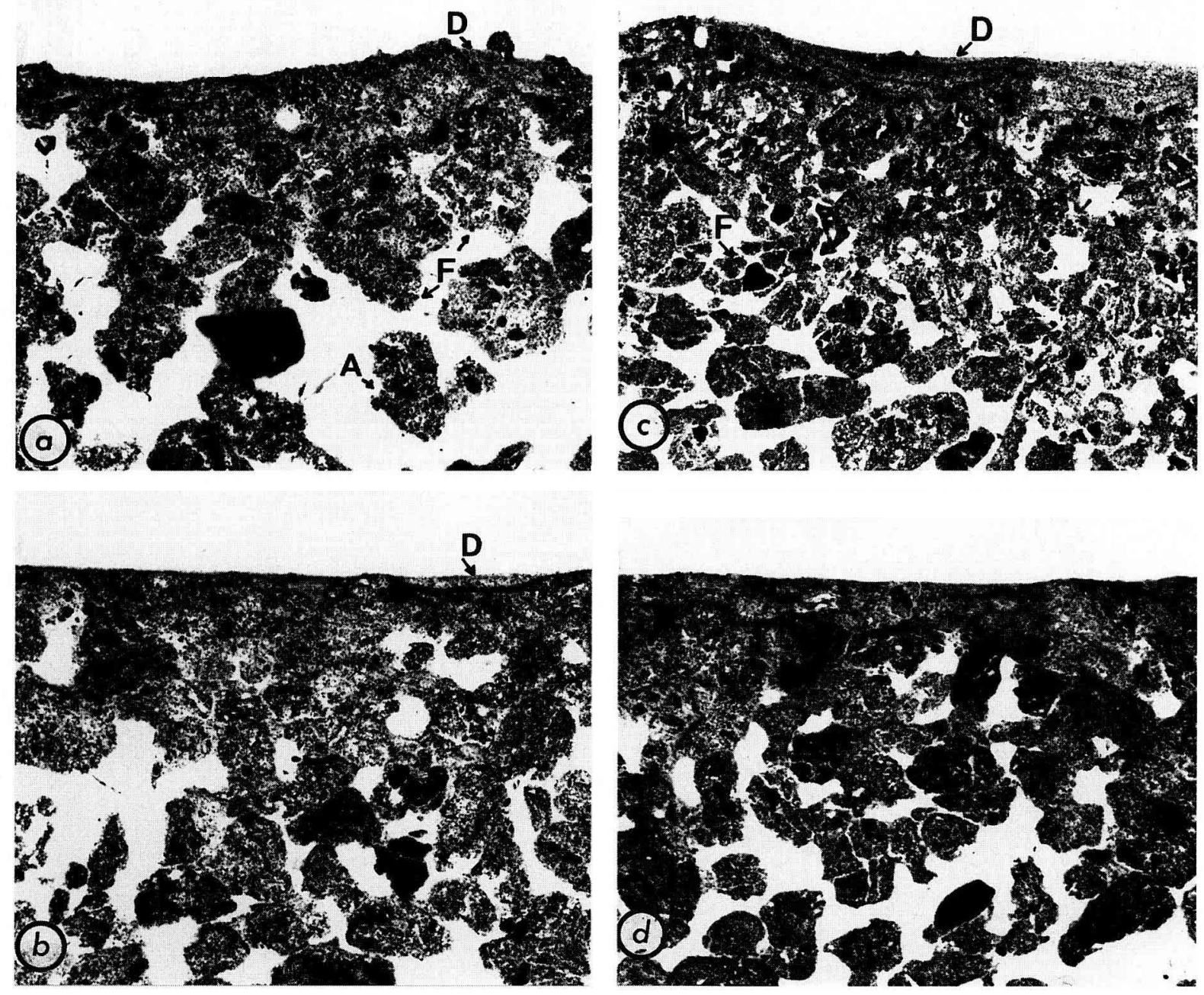

Fig 3. Profils de croûtes fragmentaires du sol limoneux (L) obtenues avec des agrégats initialement secs ou humides : $a, c=$ après 69 $\mathrm{mm}$ de pluie ; $b, d=$ après $225 \mathrm{~mm}$ de pluie; $\mathrm{F}=$ fragments d'agrégats, $\mathrm{D}=$ dépôts, $\mathrm{A}=$ agrégats. 
Pour les sols secs, la rapidité de l'apparition et de l'extension des surfaces encroûtées est en accord avec la brutalité du phénomène d'éclatement qui provoque la fragmentation, source de l'abaissement de l'infiltrabilité. Pour les sols initialement humides, la fragmentation reste encore possible sous l'action des contraintes développées à l'impact des gouttes. C'est la cohésion propre aux agrégats qui intervient ici. Le fait que la teneur en argile détermine la cohésion propre aux agrégats (Guérif, 1988) explique que la fragmentation soit observée sur le sol L-humide. Sur AL-humide, l'arrondissement des agrégats suggère la prépondérance du phénomène d'abrasion. Les particules fines doivent colmater l'espace inter-agrégats pour provoquer un abaissement de l'infiltrabilité. Ce colmatage est vraisemblablement lent puisque la formation de cratère est ici tardive.
Dans le cadre des pluies à grosses gouttes mises en jeu ici, la formation de cratère apparaît comme un indice de l'abaissement de l'infiltrabilité. C'est de manière indirecte que les facteurs de résistance des agrégats à la pluie déterminent la quantité de pluie nécessaire à leur apparition.

\section{Profil des croûtes}

Les images de section de la couche d'agrégats montrent toutes qu'une zone encroûtée repose sur un lit d'agrégats encore bien individualisés (figs 3, 4). Pour L-sec, L-humide et AL-sec, la croûte apparaît constituée de fragments et l'ensemble de ces croûtes sera qualifié ici de croûte fragmentaire. L'espace poral structural ménagé entre les fragments est particulièrement visible
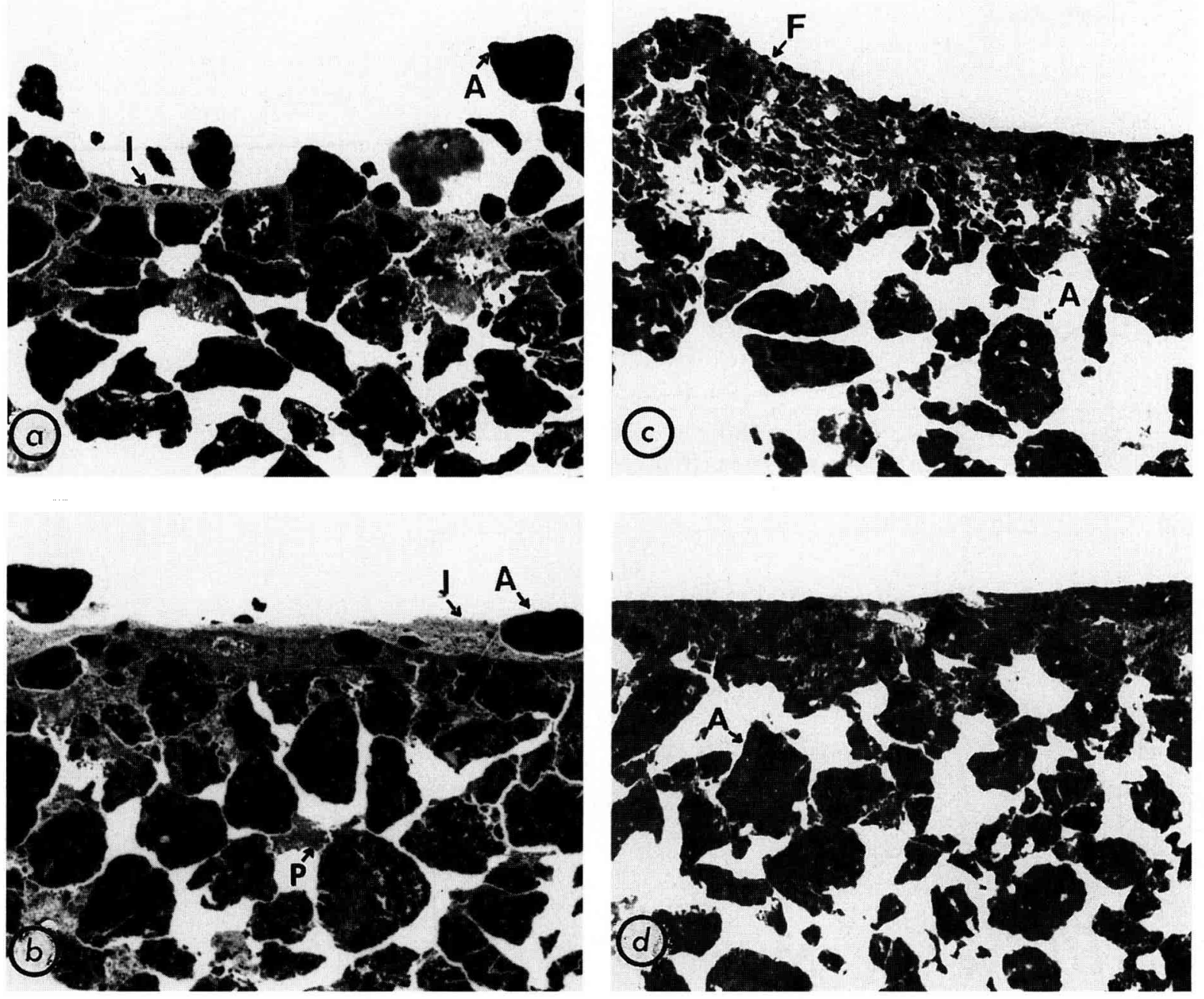

Fig 4. Profils de croûtes du sol argilo-limoneux $(\mathrm{AL})$ : a et $b$ croûtes d'illuviation obtenues en condition initiale humide ; $c$ et $d$ croûtes fragmentaires formées en condition initiale sèche : $a, c=$ après $69 \mathrm{~mm}$ de pluie ; $b, d=$ après $225 \mathrm{~mm}$ de pluie ; I= particules illuviées ; $P=$ pont de particules $; F=$ fragments d'agrégats, $D=$ dépôts, $A=$ agrégats. 
après $69 \mathrm{~mm}$ de pluie. La surface de la croûte est ondulée et montre des zones discontinues de dépôt. Après $225 \mathrm{~mm}$ de pluie, les fragments sont peu discernables, la croûte s'apparente au type coalescent décrit par Valentin et Bresson (1992). La surface de ces croûtes est plane et de minces zones de dépôt y sont parfois visibles.

Dans le cas d'AL-humide, on observe le développement d'une croûte que l'on appelera croûte d'illuviation (fig 4). En effet, après $69 \mathrm{~mm}$ de pluie, la zone encroûtée est formée par des agrégats en arrangement serré. L'espace poral structural résultant de cet assemblage est occupé par une phase claire. La ressemblance étroite avec les images fournies par Bresson et Cadot (1992) permet d'avancer qu'il s'agit de particules du squelette du sol AL. Cette croûte d'illuviation repose sur des agrégats bien individualisés. À la surface, le relief est formé par des paquets d'agrégats libres, arrondis par rapport aux agrégats visibles à la base de la section. Après $225 \mathrm{~mm}$ de pluie, la surface est plane et de rares agrégats libres y persistent. Au niveau de la croûte d'illuviation on constate que des particules du squelette ont migré en profondeur et formé des ponts inter-agrégats pouvant affecter une forme de ménisque. Enfin des dépôts foncés discontinus (non visibles sur les images) ont été notés au contact entre agrégats et sable. Ces dépôts correspondent aux revêtements argileux observés par Bresson et Cadot (1992).

En conclusion, les caractères présentés par les sections confirment les types de désagrégation observés sous pluie.
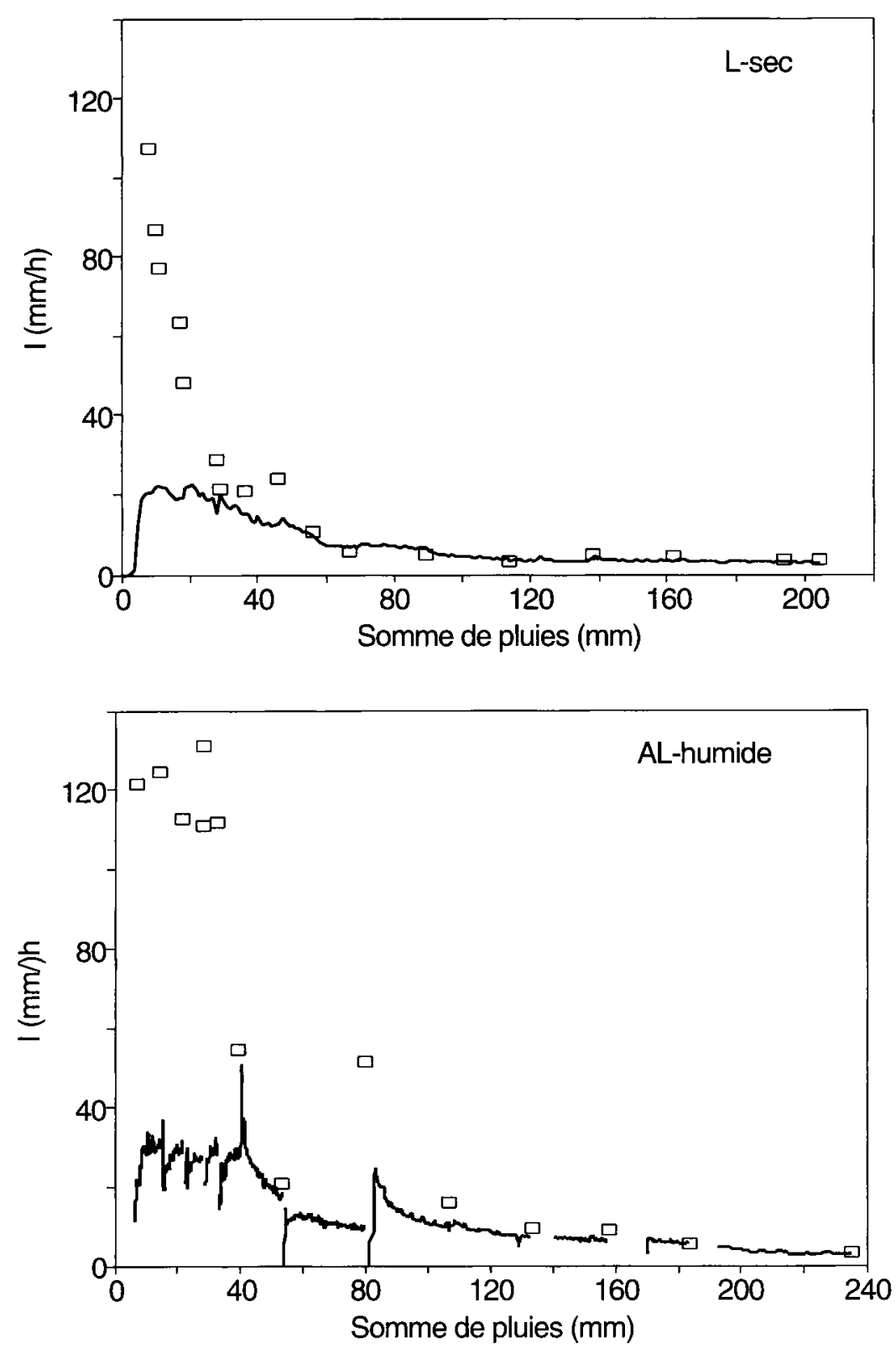

Fig 5. Exemples d'infiltrations mesurées sous pluies (ligne) et sous apport d'eau important (carré blanc), avec un état initial sec ou humide. $L$ : sol limoneux ; $A L$ : sol argilo-limoneux. 


\section{Régime d'infiltration}

Considérons tout d'abord 2 exemples de mesures d'infiltration obtenues sous pluie et sous apport d'eau important (fig 5). Les valeurs d'infiltration mesurées sous apport d'eau important sont cohérentes avec celles obtenues au cours des pluies. Elles sont en effet supérieures à celles mesurées sous pluie avant que le flaquage se manifeste, puis leurs deviennent comparables après flaquage. C'est avant ce flaquage que la valeur de l'infiltration sous apport d'eau caractérise l'infiltrabilité du système limitant formé en surface, à partir du moment où elle devient inférieure à l'intensité de l'apport. Ainsi, l'apport d'eau important permet de mesurer le niveau de l'infiltrabilité avant que cela ne devienne possible sous pluie. II apparaît que la part la plus forte de la réduction d'infiltrabilité se produit effectivement avant l'occurrence du premier flaquage (fig 5).

Les écarts observés dans les mesures d'infiltrabilité sont liés à un départ d'air. Les conditions expérimentales (agrégats disposés sur un sable saturé en eau, succion constante) imposaient la présence d'air dans l'espace inter-agrégat. Cet air pouvait être alors piégé lors des premières pluies et mis sous pression lors du développement des ménisques et de la pénétration de l'eau (Touma et Vachaud, 1984). Une expérience de vérification a confirmé que des bulles d'air apparaissaient après le flaquage, provoquant un soulèvement localisé de la croûte. Elle a aussi montré que l'apparition de bulles était synchrone avec l'accroissement temporaire du régime d'infiltration.
Sur la courbe d'infiltration sous pluies avant un premier flaquage relative à AL-humide, le régime d'équilibre n'est pas immédiatement atteint dès qu'une pluie reprend (fig 5). À l'arrêt de la pluie, une partie de l'espace poral se désature sous la succion imposée puis se sature lors d'une nouvelle pluie. Ce phénomène n'est pas aussi apparent pour le sol L-sec. Ces comportements différents vont dans le sens du maintien d'un espace poral inter-agrégats pour AL-humide alors qu'une croûte continue existe déjà à la surface pour L-sec.

\section{Comparaison des régimes d'infiltrabilité}

Afin de pouvoir comparer les régimes d'infiltrabilité, on les a représentés de manière synthétique par des courbes d'ajustement ayant la forme de la relation empirique de Horton (Lafforgue et Casenave, 1980) :

$$
\hat{l}_{(p)}=I_{n}+\left(I_{O}-I_{n}\right) * \mathrm{e}^{-\alpha * p}
$$

où $\hat{l}_{(p)}$ est l'infiltrabilité estimée ; $p$, la quantité de pluie reçue. Les paramètres d'ajustement sont l'infiltrabilité finale $I_{n}$, l'infiltrabilité initiale $I_{0}$ et le coefficient $\alpha$.

Les courbes d'infiltrabilité obtenues (fig 6) présentent toutes une phase marquée de réduction de l'infiltrabilité, suivie d'une phase finale où l'infiltrabilité varie faiblement. Cette succession est le plus souvent constatée dans la littérature pour tout un ensemble de sols, à énergie cinétique voisine ou encore 10 fois plus faible que celle mise en jeu ici (Keren, 1989). Comme le suggère le terme $I_{0}$, il faudra aussi considérer

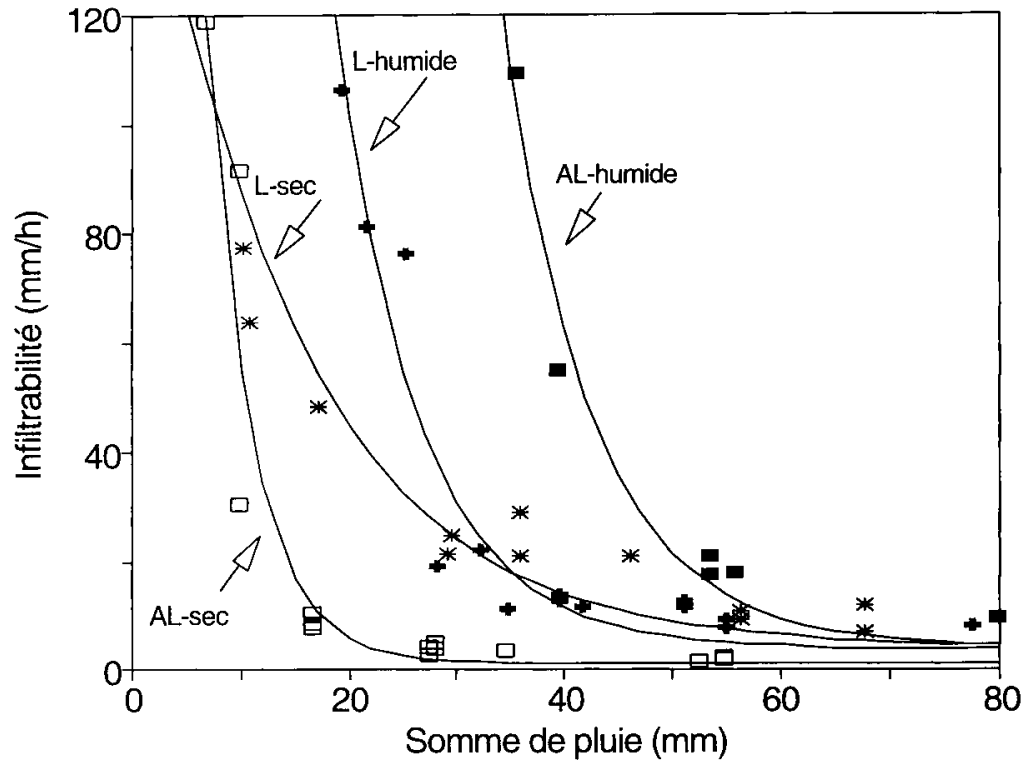

Fig 6. Effets de la teneur en eau initiale sur l'apparition et le déroulement de la phase de forte décroissance de l'infiltrabilité pour les sols limoneux (L) et argilo-limoneux (AL). 


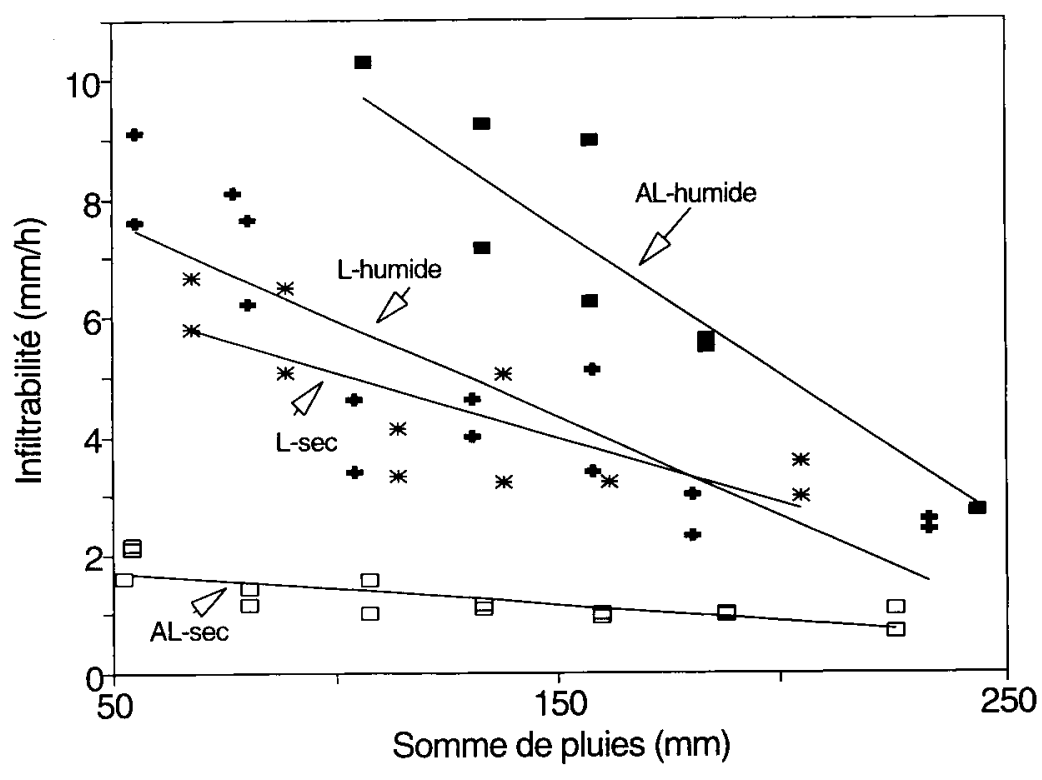

Fig 7. Phase finale d'infiltrabilité des sols limoneux (L) et argilo-limoneux $(A L)$ initialement secs ou humides. qu'une phase initiale précède la réduction d'infiltrabilité.

La différence introduite par l'état hydrique initial se traduit essentiellement par un décalage des phases de réduction. L'état humide introduit un retard à la baisse d'infiltrabilité par rapport à l'état sec. II y a donc bien une influence de la résistance propre des agrégats à l'action de la pluie, sur l'abaissement de l'infiltrabilité de la couche. Cette décroissance intervient pendant le développement de la croûte pour les sols secs et pour L-humide (fig 2). Pour L-humide en particulier, le décalage de la courbe d'infiltrabilité s'accorde avec le retard à l'encroûtement. Pour ces sols où la fragmentation des agrégats a été observée, cette phase de décroissance marquée s'achève avec l'extension complète de la croûte. II y a dans ce cas simultanéité entre l'extension de la croûte et l'abaissement marqué de l'infiltrabilité. Pour AL-humide, la baisse de l'infiltrabilité se manifeste avant que n'apparaissent les premiers signes d'encroûtement à la surface. Ici, l'initiation de l'encroûtement superficiel sur ALhumide accompagne la fin de la phase de réduction d'infiltrabilité. Ceci s'accorde bien avec le fait que les premiers cratères d'impact de gouttes aient été observés peu avant cette initiation. La phase majeure de réduction est donc provoquée ici par le colmatage des pores inter-agrégats. Ce colmatage échappe à l'observation de surface. L'encroûtement qui a été observé sur AL-humide ne représente que l'achèvement du remplissage des espaces interagrégats en surface ainsi que la disparition des agrégats restés libres à la surface. Cet encroûtement coïncide ici avec la phase finale. En définitive, la réduction marquée d'infiltrabilité est une phase complexe et peu homogène, où les surfaces encroûtées ou colmatées se développent pendant que les modifications physiques se poursuivent sur les surfaces déjà dégradées.

La phase initiale d'évolution de l'infiltrabilité se situe apparemment à une valeur supérieure à $120 \mathrm{~mm} / \mathrm{h}$ et n'a pas pu être mesurée. À cet égard, les valeurs obtenues pour $I_{0}$ n'ont pas de signification physique. En effet elles varient d'un facteur 20 entre $L$-sec et $A L$-humide bien que dans les 2 cas il s'agisse d'agrégats de même calibre mis en place dans les mêmes conditions et devant donc présenter des niveaux voisins d'infiltrabilité initiale. Une phase de faible réduction de l'infiltrabilité initiale doit plutôt précéder la phase d'abaissement marqué, comme le suggèrent Baumhardt et al (1990).

La phase finale d'infiltrabilité correspond à l'évolution d'une surface entièrement encroûtée. Les causes de cette évolution peuvent être recherchées dans les modifications de l'état physique de la croûte elle-même. L'infiltrabilité varie linéairement avec la somme de pluie (fig 7) mais le classement des courbes ne reflète pas tout à fait celui des constitutions. Ainsi l'infiltrabilité reste toujours la plus faible sur AL-sec et elle présente des valeurs voisines pour L-sec et L-humide. En revanche, $A L$-humide conserve l'infiltrabilité la plus élevée, mais cette infiltrabilité s'abaisse vers le niveau d'infiltrabilité commun à L-sec et L-humide. Ces niveaux d'infiltrabilité sont du même ordre de grandeur que ceux indiqués dans la littérature. Mais des effets contradictoires apparaissent aussi en fonction de la constitution. Ainsi, un taux élevé en sable ou en argile peut conférer une infiltrabili- 
té plus élevée qu'un taux important en limon (Ben-Hur et Shainberg, 1989), mais, à même teneur en argile, un sol à dominante de sable peut avoir la même infiltrabilité qu'un sol à dominante de limon (Keren, 1989).

Ces exemples joints aux différences d'infiltrabilité notées entre AL-sec et AL-humide suggèrent que le régime final d'infiltrabilité peut correspondre à des états physiques différents. C'est pour répondre à ce point que l'espace poral des croûtes a été analysé.

\section{Porosimétrie des croûtes}

Le volume total de mercure injecté est plus grand dans les croûtes que dans les agrégats. La différence des volumes représente une estimation du volume structural dans les croûtes (tableau II). Le volume structural se réduit sous l'effet des pluies. II est aussi plus faible sur les croûtes L-humide mais cet effet de l'état hydrique initial n'apparaît pas sur les croûtes AL. On a porté sur le même tableau les valeurs du volume structural des croûtes aux différents équilibres de potentiel. Elles sont obtenues par différence entre le volume massique de la croûte et celui de l'agrégat. Le volume structural mis en évidence par porosimétrie est donc conservé à l'état humide. II en est de même pour son ordre de grandeur (la moyenne des valeurs fournies par l'une et l'autre méthode est voisine de $1,6 \mathrm{~cm}^{3} / 100 \mathrm{~g}$ ) et pour les effets de la quantité de pluie reçue et de l'état hydrique initial.

Les courbes de distribution précisent la gamme des diamètres concernée par ces différences d'intrusion. Tout d'abord, les courbes relatives aux agrégats présentent toutes un pic marquant l'intrusion dans les pores lacunaires (fig 8).
Cette intrusion commence vers $10 \mu \mathrm{m}$ pour les agrégats du sol $\mathrm{L}$ et vers $5 \mu \mathrm{m}$ pour $\mathrm{AL}$. La teneur plus forte en argile de ce dernier se traduit par un début d'intrusion dans les pores de l'argile, reconnaissable après $0,05 \mu \mathrm{m}$. Pour les croûtes, les courbes de distribution ne diffèrent de celles des agrégats qu'en début d'intrusion. Dans les croûtes L, l'intrusion débute comme sur les agrégats au voisinage de $10 \mu \mathrm{m}$, mais les quantités introduites sont plus fortes entre 10 et $2 \mu \mathrm{m}$. Sur les croûtes AL, l'intrusion débute au voisinage de $10 \mu \mathrm{m}$, donc par des diamètres de pore supérieurs à ceux des agrégats $\mathrm{AL}$.

Les différences dans les diamètres d'intrusion entre croûte et agrégat peuvent s'interpréter en tenant compte des différences d'origine et d'organisation des éléments constituant les croûtes, déjà mises en évidence en particulier sur les images de profils de croûtes (figs 3,4 ). Les schémas d'organisation résultant pour les croûtes sont présentés à la figure 9 . Pour les croûtes fragmentaires, 2 étapes de la modification des croûtes sous l'action des pluies sont illustrées. Dans les croûtes AL-sec, l'intrusion dans l'espace poral structural s'effectue par des pores de taille supérieure à celle des pores lacunaires. Les pores structuraux constituent encore un réseau de pores grossiers interconnectés. Avec les croûtes $L$, l'accès aux pores structuraux n'est déjà plus différenciable de l'accès aux lacunes.

Ces résultats illustrent le phénomène de compaction par les gouttes de pluie suggéré par McIntyre (1958). Ils peuvent être rapprochés de ceux obtenus par Cousin et al (1994) sur des massifs d'agrégats compactés. Ces auteurs montrent que l'accès aux pores structuraux est d'abord direct sur les massifs les moins compac-

Tableau II. Volume structural des croûtes $\left(\mathrm{cm}^{3} / 100 \mathrm{~g}\right.$ ) estimé par porosimétrie au mercure (en gras) et par mesure de volume massique entre les potentiels hydriques de $-0,1 \mathrm{~m}$ à $-10 \mathrm{~m}$, pour les différents états hydriques inititaux et quantités de pluie reçues.

Pluie

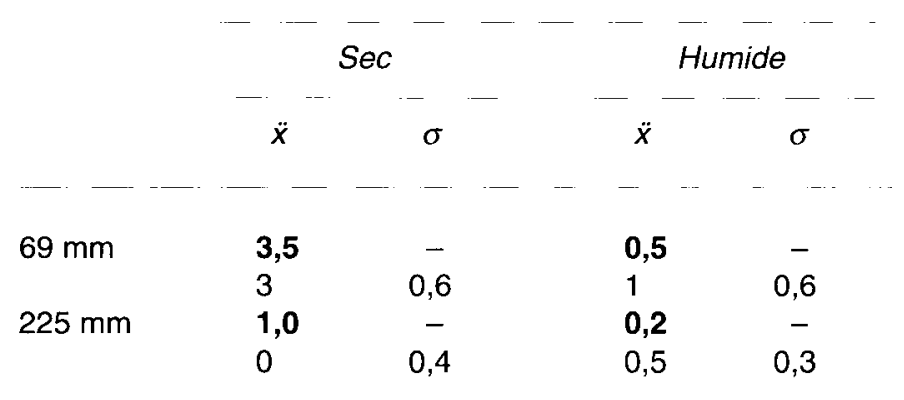

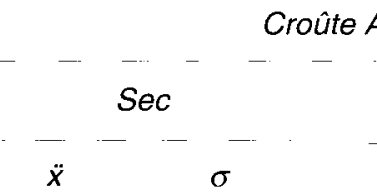

2,4

2

1,2

3
Croûte $A L$

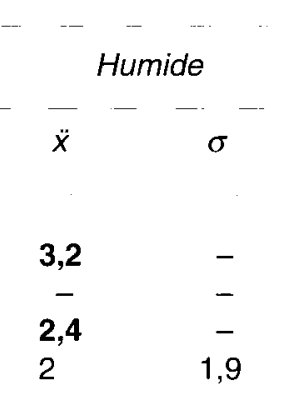



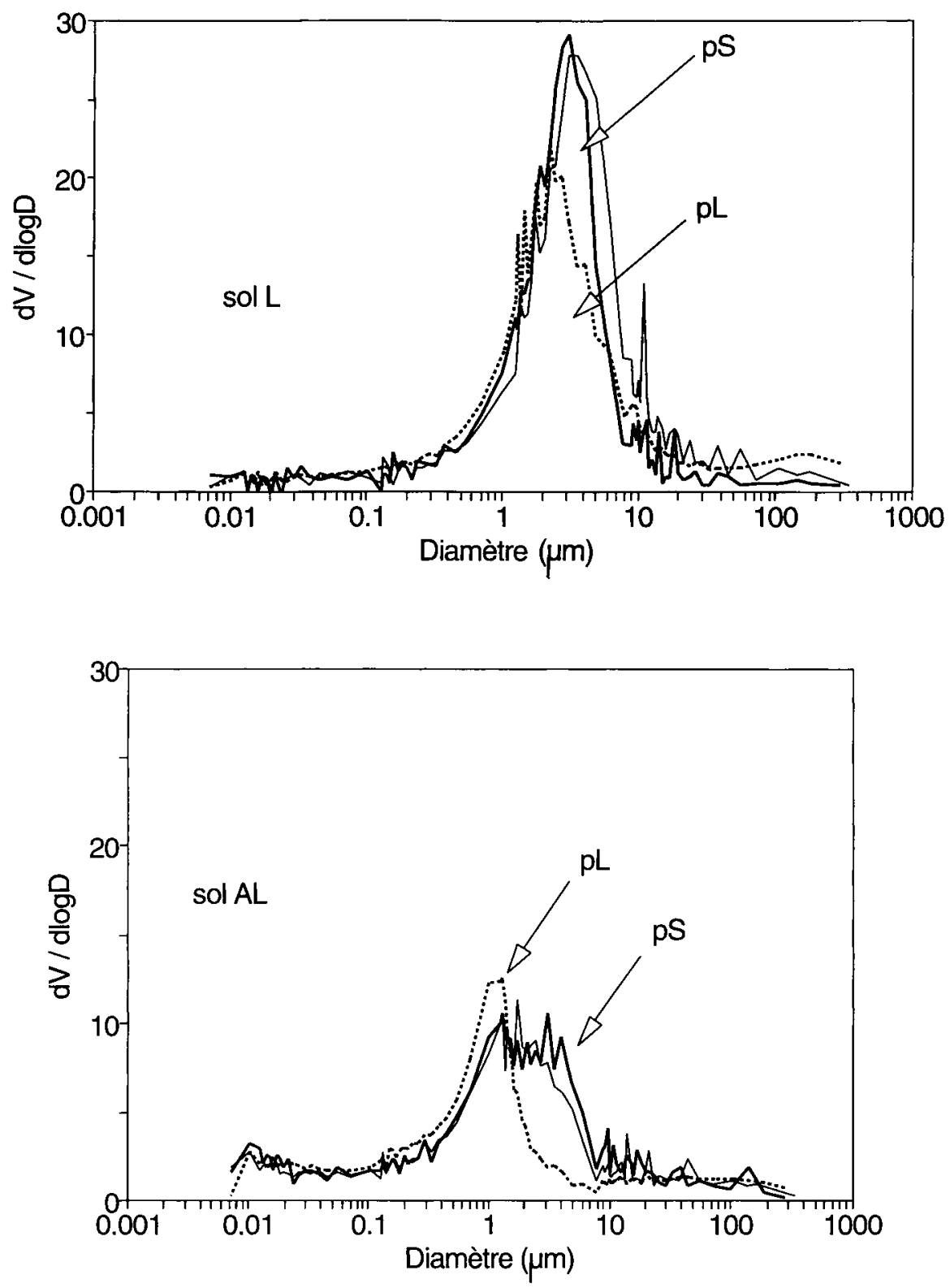

Fig 8. Exemples de courbes de distribution obtenues par porosimétrie au mercure sur des agrégats ou sur des croûtes prélevées après 225 $\mathrm{mm}$ de pluie, pour 2 états hydriques (trait pointillé : agrégats ; trait gras : humide ; trait fin : sec). $L$ : sol limoneux ; $A L$ : sol argilo-limoneux. L'intrusion de mercure dans les pores est notée $\mathrm{pL}$ pour les pores lacunaires, pS pour les pores structuraux.

tés puis s'effectue par l'intermédiaire des pores lacunaires sur les massifs les plus compactés.

Pour les croûtes d'illuviation, la porosité structurale a son origine dans l'espace inter-agrégat. L'accès à cet espace s'effectue au travers des pores d'assemblage des particules du squelette qui l'encombrent partiellement. Ces pores d'accès sont plus grossiers que les pores texturaux des agrégats. En effet, l'étude de mélanges d'argile et de squelette a montré qu'aux teneurs en argile supérieures à 20 ou $25 \%$, le diamètre modal des pores lacunaires devient inférieur à celui du squelette sans argile (Fiès, 1992).

Par rapport aux profils de croûtes (figs 3,4 ), la porosimétrie apporte une information sur le degré d'interconnexion des pores structuraux. Cependant, comme l'illustre la grande ressem- blance des courbes de distribution des croûtes $\mathrm{AL}$, cette méthode ne permet pas de différencier la croûte fragmentaire de la croûte d'illuviation. En fait, l'interprétation doit aussi s'appuyer sur des approches indépendantes, comme l'observation de sections. Un autre exemple en sera fourni par l'étude de la rétention en eau des croûtes.

Vis-à-vis de l'infiltrabilité finale des croûtes, la ressemblance entre L-sec et L-humide s'explique par celle de la conformation de leur espace poral. II en va différemment en revanche pour les croûtes AL. Si la présence d'un espace poral structural directement accessible à l'eau rend compte du niveau d'infiltrabilité des croûtes d'illuviation $\mathrm{AL}$, elle est en contradiction avec le faible niveau d'infiltrabilité des croûtes fragmentaires AL. 


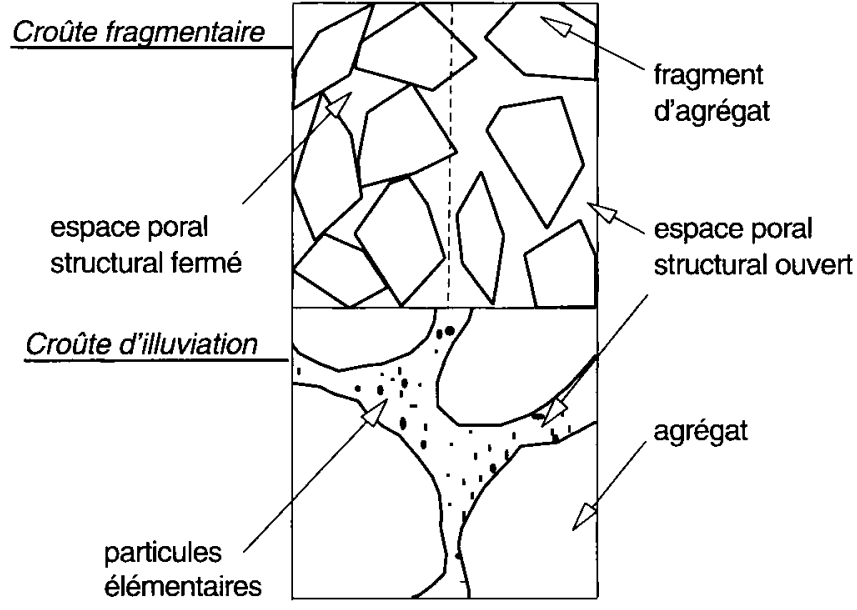

Fig 9. Représentations de l'organisation des croûtes fragmentaires et d'illuviation.

\section{Rétention en eau des croûtes}

Par rapport à la porosimétrie au mercure, l'étude de la rétention en eau a l'avantage de caractériser les croûtes dans des états voisins de ceux correspondant à leur fonctionnement sous pluie. Pour le sol $L$, la teneur pondérale en eau des croûtes $W_{c}$ est généralement supérieure à la teneur pondérale en eau des agrégats $W_{a g}$ pour les potentiels allant de $-0,1$ à $-5 \mathrm{~m}$. Dans cet intervalle, la moyenne des écarts $W_{c}-W_{\text {ag }}$ est la plus forte sur les croûtes après $69 \mathrm{~mm}$ de pluie et représente $1 \%$ pour la croûte $L-\sec$ et $4 \%$ pour la croûte L-humide. Après $225 \mathrm{~mm}$ de pluie, la moyenne des écarts ne représente plus que $0,4 \%$ pour les 2 états hydriques confondus. Enfin, au potentiel de $-10 \mathrm{~m}$, les croûtes $L$ retiennent toutes un peu moins d'eau que les agrégats, la moyenne des écarts étant de $-0,6 \%$.

Pour le sol AL, 2 types de résultats sont obtenus dans l'intervalle $-0,1$ à $-10 \mathrm{~m}$. Les croûtes AL-sec retiennent plus d'eau, la moyenne des écarts étant de $0,8 \%$ avec les agrégats, sans effet marqué de la quantité de pluie reçue. Sur la croûte AL-humide, après $225 \mathrm{~mm}$ de pluie, cette moyenne est de $-0,8 \%$ (il faut noter qu'aucune mesure n'a été possible sur les croûtes AL-humide obtenues après $69 \mathrm{~mm}$ de pluie, du fait de leur fragilité).

Ainsi, la rétention d'un excès d'eau par rapport aux agrégats est le fait des croûtes $L$ et $A L$ quand elles sont fragmentaires. Cet excès confirme la présence d'un espace poral structural. II suggère aussi que ces pores restent saturés pour assurer le transfert de l'eau, en particulier pour la succion de $-0,4 \mathrm{~m}$ imposée dans le dispositif. Pour le vérifier, le taux de saturation volumique des croûtes est comparé à celui des agrégats. Ce taux a été calculé en utilisant les valeurs de masse volumiques des échantillons mesurées aux différents équilibres de potentiel.

Le taux de saturation des croûtes $L$ se confond avec celui des agrégats jusqu'à $-5 \mathrm{~m}$ de succion (fig 10), ce qui suggère bien que ce sont les pores texturaux des croûtes qui participent à la rétention de l'eau au cours des pluies. Le taux de saturation ne devient inférieur à celui des agrégats qu'au potentiel de $-10 \mathrm{~m}$, ce qui correspondrait à la vidange des pores structuraux par les pores lacunaires les plus grossiers. Au cours des pluies, les croûtes $L$ restent donc saturées et ce sont les caractéristiques des pores texturaux qui limitent le transfert de l'eau.

Les croûtes $A L$, quelle que soit leur nature, apparaissent plus désaturées que les agrégats pour les potentiels inférieurs à $-0,1 \mathrm{~m}$, la désaturation étant la plus marquée avec AL-humide après $225 \mathrm{~mm}$ de pluie (fig 10). Ces résultats confirment certes le fait que les pores structuraux de ces croûtes sont plus grossiers que les pores lacunaires des agrégats. Mais, en définitive, la différence d'infiltrabilité des croûtes fragmentaires $A L$ et des croûtes d'illuviation $A L$ ne semble pas liée ici non plus à des différences de conformation des pores. En particulier ces croûtes doivent partiellement se désaturer entre chaque pluie, quand la succion imposée de $-0,4 \mathrm{~m}$ peut se traduire jusqu'à la surface. Pour expliquer leur différence d'infiltrabilité il faudrait alors admettre que, lors de la reprise de la pluie, de l'air resterait piégé dans les pores structuraux des croûtes fragmentaires $A L$, ce qui les empêcherait d'être fonctionnels vis-à-vis du transfert de l'eau. Dans les croûtes d'illuviation AL, ce piégeage d'air serait moins important et moins efficace.

\section{CONCLUSION}

Le processus d'encroûtement d'une surface constituée d'agrégats de même dimension apparaît comme un sous-ensemble du processus général de l'encroûtement d'un sol travaillé démontré par Boiffin (1985). Son existence est liée à la mise en ceuvre de gouttes de fort diamètre, qui accuse le mode discret de l'action des pluies par rapport à des pluies de granulométrie plus étalée. 

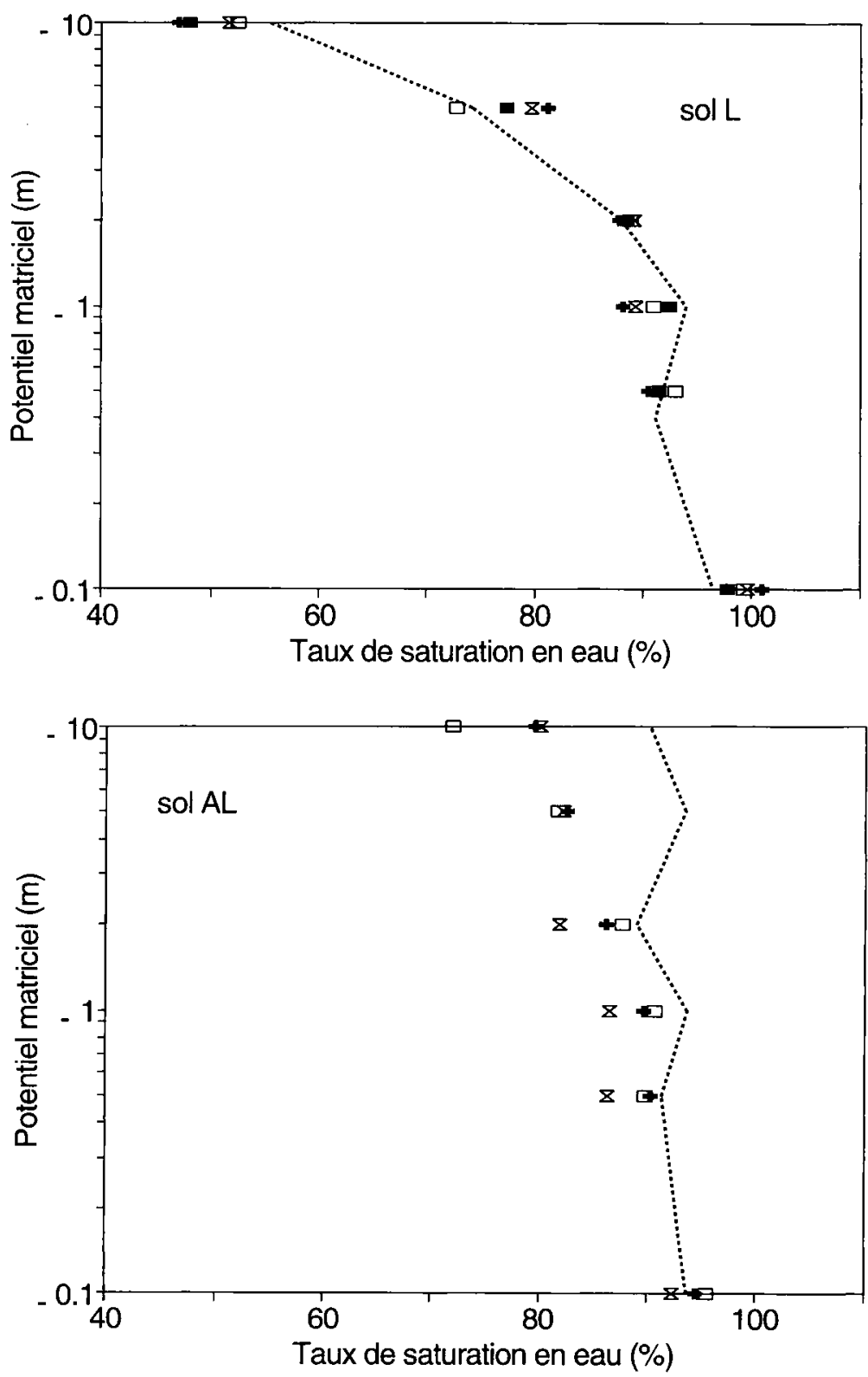

Fig 10. Taux de saturation en eau des croûtes et des agrégats, aux équilibres de potentiel compris entre $-0,1$ et $-10 \mathrm{~m}$. État initia humide : carré plein $=69 \mathrm{~mm}$ de pluie ; carré vide $=225 \mathrm{~mm}$ de pluie. État initial sec : croix $=69$ $\mathrm{mm}$ de pluie ; sablier $=225 \mathrm{~mm}$ de pluie. Agrégat $=$ ligne pointillée

La mesure de l'infiltrabilité avant flaquage sous pluie montre que la phase de réduction importante de l'infiltrabilité est assez brutale. Une évolution plus lente serait observée dans le cas de pluies à gouttes plus fines et de moindre énergie cinétique (Keren, 1989). L'état hydrique initial influe sur la quantité de pluie qui initie la diminution d'infiltrabilité. Cette quantité est plus élevée quand le sol est initialement humide. L'intérêt des millimètres de pluie ainsi gagnés par rapport à un état sec initial peut s'évaluer en fonction des caractéristiques climatiques de la période suivant par exemple une date de semis: dans les cas favorables, ils permettront que la levée s'effectue en l'absence d'obstacles mécaniques (Arndt, 1965).

Les conditions permettant d'obtenir un état hydrique initial humide sans dégradation impor- tante sont celles de pluies fines de faible intensité. Dans le cadre général d'une prévision du ruissellement sur la base d'une cinétique de dégradation de l'état de surface, il conviendrait alors d'ajouter à l'effet non dégradant des épisodes pluvieux de faible énergie cinétique (Boiffin, 1985), un effet protecteur vis-à-vis de pluies ultérieures plus agressives.

La phase finale où l'infiltrabilité diminue lentement recouvre des conformations différentes de l'espace poral des croûtes. Le couplage d'observations et de méthodes de mesure indépendantes a permis d'aboutir à des schémas cohérents de son organisation pour les croûtes fragmentaires et les croûtes d'illuviation. Le trait principal est que la présence d'un espace poral structural n'assure pas nécessairement un 
niveau plus élevé d'infiltrabilité puisqu'il peut soit ne fonctionner que par l'intermédiaire des pores texturaux, soit ne pas être fonctionnel parce qu'insaturé sous pluie.

\section{REMERCIEMENTS}

G Monnier a initié ce travail et les auteurs ont largement bénéficié des idées et du soutien qu'il leur a offerts. M Bourlet et $Y$ Duval ont fourni les sols et $C$ Jeandet a réalisé les mesures.

\section{RÉFÉRENCES}

Al-Durrah M, Bradford JM (1982) The mechanism of raindrop splash on soil surfaces. Soil Sci Soc Am J 46, 1086-1090

Arndt $W$ (1965) The nature of the mechanical impedance to seedlings by soil surface seals. Aust $J$ Soil Res 3, 45-54

Baumhardt RL, Römkens MJM, Whisler FD (1990) Modeling infiltration into a sealing soil. Water Resour Res 26, 2497-2505

Ben-Hur M, Shainberg I (1989) Clay, calcium carbonate and sodium polymetaphosphate interactions in soil seal formation. Soil Sci Soc Am J 53, 242-247

Boiffin J (1984) La dégradation structurale des couches superficielles du sol sous l'action des pluies. Thèse docteur-ingérieur, Paris INA-PG, 320 $p+$ annexes

Boiffin J (1985) Stages and time-dependency of soil crusting in situ. In : Assessment of Soil Surface Crusting. Proc Symposium ISSS AISS IBG Ghent (BEL) 1985 (F Callebaut, D Gabriels, M De Boodt, eds), State University of Ghent, Ghent, 91-98

Boiffin J, Guérif J, Stengel P (1990) Les processus d'évolution de l'état structural du sol : quelques exemples d'études expérimentales récentes. In : $\mathrm{La}$ structure du sol et son évolution, Laon (FRA) 1990 (INRA Paris, ed), Les Colloques de I'INRA, 59, 37-69

Boiffin J, Monnier G (1985) Infiltration rate as affected by soil surface crusting caused by rainfall. In : Assessment of Soil Surface Crusting. Proc Symposium ISSS AISS IBG Ghent (BEL) 1985 (F Callebaut, D Gabriels, M De Boodt, eds), State University of Ghent, Ghent, 210-217

Bresson LM, Cadot L (1992) Illuviation and structural crust formation on loamy temperate soils. Soil Sci Soc Am J 56, 1565-1570

Bruand A, Prost R (1987) Effect of water content on the fabric of a soil material: an experiential approach. J Soil Sci 38, 461-472

Cernuda CF, Smith RM, Vicente-Chandler J (1954) Influence of initial soil moisture condition on resistance of macroaggregates to slaking and to waterdrop impact. Soil Sci 77, 19-27
Childs EC (1969) An Introduction to the Physical Basis of Soil Water Phenomena. Wiley-Interscience, London

Cousin I, Le Lay C, Bruand A (1994) Évolution de la porosité et formation de pores structuraux reliques dans un sol argilo-limoneux soumis à un compactage. C R Acad Sci Ser II 319, 797-804

De Roo APJ, Riezebos HT (1992) Infiltration experiments on loess soils and their implications for modeling surface runoff and soil erosion. Catena 19, 221-239

Epema GF, Riezebos HT (1983) Fall velocity of waterdrops at different heights as a factor influencing erosivity of simulated rain. In : Rainfall Simulation, Runoff and Soil Erosion. Catena supplement 4 (Jan de Ploey, ed), Braunschweig, 1-16

Fiès JC (1992) Analysis of soil textural porosity relative to skeleton particles size, using mercury porosimetry. Soil Sci Soc Am J 56, 1062-1067

Fiès JC, Bruand A (1990) Textural porosity analysis of a silty clay soil using pore volume balance estimation, mercury porosimetry and quantified backscattered electron scanning image (BESI). Geoderma 47, 209-219

Fiès JC, Zimmer D (1981) Étude expérimentale de modifications de l'assemblage textural d'un matériau sablo-argileux sous l'effet de pressions. Bull Groupe Fr Humidimétrie Neutronique 12, 39-54

Freebairn DM, Gupta SC, Rawls WJ (1991) Influence of aggregate size and microrelief on development of surface soil crusts. Soil Sci Soc Am J 55, 188-195

Govers G, Poesen J (1985) A field scale study of surface sealing and compaction on loam and sandy loam soils. Part I. Spatial variability of soil surface sealing and crusting. In : Assessment of Soil Surface Crusting. Proc Symposium ISSS AISS IBG Ghent (BEL) 1985 (F Callebaut, D Gabriels, M De Boodt, eds), State University of Ghent, Ghent, 171182

Guérif J (1988) Résistance en traction des agrégats terreux : influence de la texture, de la matière organique et de la teneur en eau. agronomie 8, 375-386

Hartmann C, Tessier D, Wilding LP (1992) Simultaneous use of transmitted and incident ultraviolet light in describing soil microfabrics. Soil Sci Soc Am J 56, 1867-1870

Hénin S, Monnier G (1956) Évaluation de la stabilité de la structure du sol. In : VI Congrès Int Dci Sol AISS Paris 1956, vol B, 49-52

Kheyrabi D, Monnier G (1968) Étude expérimentale de l'influence de la composition granulométrique des terres sur leur stabilité structurale. Ann Agron 19, 129-152

Keren R (1989) Water drop kinetic energy effect onwater infiltration in calcium and magnesium soils. Soil Sci Soc Am J 53, 1624-1628

Lafforgue A, Casenave A (1980) Premiers résultats obtenus en zone tropicale sur les modalités de transfert pluie-débit par l'emploi de simulateurs de pluie. Houille Blanche 4-5, 243-250 
Le Bissonnais $Y$ (1988) Comportement d'agrégats terreux soumis à l'action de l'eau : analyse des mécanismes de désagrégation. agronomie 8, 915-924

Le Bissonnais $Y$, Bruand A, Jamagne M (1989) Laboratory experimental study of soil crusting: relation between aggregate breakdown mechanisms and crust structure. Catena 16, 377-392

Le Souder C, Le Bissonnais Y, Robert M (1991) Influence of a mineral conditioner on the mechanisms of disaggregation and sealing of a soil surface. Soil Sci 152, 395-402

Mclntyre DS (1958) Permeability measurements of soil crusts formed by raindrop impact. Soil Sci $85,185-$ 189

Monnier G (1984) Recherches sur la stabilité structurale, exemples d'applications. In : Livre jubilaire du cinquantenaire AFES 1934/1984 (CNRS, INRA, ORSTOM, MIDIST, eds), 293-297

Monnier G, Stengel P, Fiès JC (1973) Une méthode de mesure de la densité apparente de petites agglomérats terreux. Application à l'analyse des systèmes de porosité du sol. Ann Agron 24, 533-545

Panini T, Salvador-Sanchis MP, Torri D (1993) A portable rain simulator for rough and smooth morphologies. Quaderni di Scienza del Suolo 5, 47-58

Poesen J, Ingelmo-Sanchez MP, Mücher H (1990) The hydrological response of soil surfaces to rainfall as affected by cover and position of rock fragments in the top layer. Earth Surface Processes and Landforms 15, 653-671

Renault P (1988) Theoretical studies on mercury intrusion in some networks: testing the applicability of mercury intrusion in the size characterisation of the lacunar pore space of soil samples. Transp Porous Media 3, 529-547

Sharma PP, Gupta SC (1989) Sand detachment by single raindrops of varying kinetic energy and momentum. Soil Sci Soc Am J 53, 1005-1010

Slattery MC, Bryan RB (1992) Laboratory experiments on surface seal development and its effect on interrill erosion processes. J Soil Sci 43, 517-529

Stengel P (1979) Utilisation de l'analyse des systèmes de porosité pour la caractérisation de l'état physique du sol in situ. Ann Agron 30, 27-51

Stengel P, Bourlet M (1987) Fissuration d'un sol argileux gonflant après compactage : Effet de l'humectation. In : Soil compaction and regeneration. Proc Workshop Soil Compaction: Consequences and structural regeneration processes Avignon (FRA) 1985 ( $G$ Monnier, MJ Goss, eds), Balkema, Rotterdam, 95-110

Tamari S, Gaudu JC, Simoneau T (1993) Tensiometric measurement and metastable state of water under tension. Soil Sci 156, 149-155

Touma J, Vachaud G (1984) Air and water flow in a sealed, ponded vertical soil column: experiment and model. Soil Sci 137, 181-187

Truman CC, Bradford JM, Ferris JE (1990) Antecedent water content and rainfall energy influence on soil aggregate breakdown. Soil Sci Soc Am J 54, 13851392

Valentin C, Bresson LM (1992) Morphology, genesis and classification of surface crusts in loamy and sandy soils. Geoderma 55, 225-245 